\title{
RecA-independent high-frequency deletion of recombinant cosmid DNA in Escherichia coli
}

\author{
Masahiro Ishiura, ${ }^{1 *}$ Nobuyoshi Hazumi, ${ }^{1}$ Hideo Shinagawa, ${ }^{2}$ Atsuo Nakata, ${ }^{2}$ \\ TSUYOSHI UCHIDA ${ }^{3}$ and YoshIO OKADA ${ }^{1,3}$ \\ ${ }^{1}$ Division of Cell Fusion, Department of Cell Biology, National Institute for Basic Biology, Myodaiji-cho, Okazaki, \\ Aichi 444, Japan \\ ${ }^{2,3}$ Research Institute for Microbial Diseases ${ }^{2}$ and Institute for Molecular and Cellular Biology ${ }^{3}$, Osaka University, \\ Yamada-oka, Suita, Osaka 565, Japan
}

(Received 10 July 1989; revised 6 September 1989; accepted 9 October 1989)

\begin{abstract}
Segments of DNA were deleted from recombinant cosmid DNAs during propagation in Escherichia coli hosts in liquid culture. DNAs of more than 1000 cosmids propagated in various $E$. coli hosts were analysed by agarose gel electrophoresis (AGE). The effects of vectors, insert DNAs and host genetic characters on the formation of deletions were examined. The probability of deletion and the pattern of deletion bands observed by AGE differed from clone to clone, and after extensive culture the deletion band patterns remained almost constant during further culture. Most recombinant clones eventually showed deletion during prolonged liquid culture. Mutations in the $\boldsymbol{r e c} A$ gene of $E$. coli hosts, including a deletion mutation, did not prevent deletion. Most deletions occurred in the insert portions of cosmid DNAs. Nucleotide sequence analysis of six deletion junctions in test cosmid cMB15 demonstrated that deletions occurred between two short complete direct repeats of about 4-10 bp, irrespective of whether the cosmid was propagated in a rec $A$ host or a rec ${ }^{+}$host. Some deletions occurred at the same sites either in a $\operatorname{rec} A$ host or a rec ${ }^{+}$host. These results suggest that the deletion events are mainly mediated by a $\operatorname{rec} A$-independent recombination system(s) of $E$. coli host cells.
\end{abstract}

\section{Introduction}

A cosmid vector is a plasmid vector carrying the phage $\lambda$ cos sequence, which allows cloning of large DNA fragments of up to about $50 \mathrm{~kb}$ through in vitro packaging of DNA into phage particles (for a review, see Maniatis et al., 1982). Due to its large capacity, such a vector is very useful for producing mammalian genomic libraries with a minimum number of clones and cloning genes of interest from the libraries, because many mammalian genes are more than $20 \mathrm{~kb}$ in size (for a review, see Ishiura et al., 1989a), which is the cloning capacity of the $\lambda$ vector (Maniatis et al., 1982). Some genes can be cloned without interruption into a single cosmid. However, we have encountered several difficulties in cosmid cloning. One of the most serious problems is the occurrence of

This paper is dedicated by the coauthors (M.I., N.H., H.S., A.N. and Y.O.) to the memory of the late Professor Tsuyoshi Uchida, who passed away at the age of 52, on May 3, 1989.

\footnotetext{
*Author for correspondence (electronic mail address: Bitnet ISHIURA@JPNONRI).
}

deletions in cosmid DNAs during their amplification in Escherichia coli host cells : probably because of their large size, cosmids do not seem to be stably maintained in $E$. coli hosts. These deletions have been mentioned in several papers (Chia et al., 1982; Steinmetz et al., 1982; Yelton et al., 1985), but they have not yet been extensively characterized, and the primary importance of rec $A$ mutations in $E$. coli hosts to prevent the cosmid deletion events has been presumed rather than investigated systematically (Ish-Horowicz \& Burke, 1981; Chia et al., 1982; Maniatis et al., 1982). Several mechanisms have been proposed for the various deletion events that occur in recA E. coli hosts (Brutlag et al., 1977; Collins, 1980; Lilley, 1981 ; Liu \& Lark, 1982; Collins et al., 1982; Boissy \& Astell, 1985; Saing et al., 1988). However, it has not been known whether such mechanisms are involved in the cosmid deletion events. Recently, we analysed

Abbreviations: AGE, agarose gel electrophoresis; Ap, ampicillin; bla, $\beta$-lactamase gene; $\mathrm{CHO}$ cell, Chinese hamster ovary cell; $\mathrm{LB}$, LuriaBertani broth; LB-Ap medium, LB containing $30 \mu \mathrm{g} \mathrm{Ap} \mathrm{ml}^{-1}$; LB-Tc medium, LB containing $10 \mu \mathrm{g} \mathrm{Tc} \mathrm{ml} l^{-1}$; ori, origin of replication; Tc, teTc, tetracycline hydrochloride. 
cosmid deletion events using six specific cosmids and demonstrated that four of the cosmids showed deletions during propagation in $\mathrm{rec} A$ hosts, whereas two did not show such deletions (Ishiura et al., 1989b). We have also demonstrated that $\operatorname{rec} B$ rec $C s b c B$ recJ hosts prevented the cosmid deletion events that occurred in rec $A$ hosts (Ishiura et al., 1989b). However, it is uncertain whether all cosmid deletion events are $\operatorname{rec} A$-independent and prevented by $r e c B$ rec $C s b c B$ recJ hosts. In addition, some authors have suggested the possible involvement of an insertion element in cosmid deletion events (Fernandez et al., 1986), whereas others have suggested that the use of ori of phage $\lambda$ in place of that of pBR322 as ori of the vector may reduce the frequency of the deletions (Little \& Cross, 1985).

To address the problems described above, we have investigated the effects of various vectors, insert DNAs prepared from several organisms, and host genetic backgrounds related to recombination, especially the rec $A$ gene, on the generation of deletions in cosmids during propagation in $E$. coli hosts.

\section{Methods}

Bacterial strains, media, and manipulation of DNA. The bacterial strains used in this study are listed in Table 1. The derivatives of C600 $\mathrm{r}^{-} \mathrm{m}^{-}$and ED8654 carrying a deletion mutation in the $\mathrm{rec} A$ gene were constructed by Pl transduction (Miller, 1972), using JC10289 and Pl vir as donor and transducing phage, respectively. Transductants were selected on LB agar plates (see below) containing $10 \mu \mathrm{g} \mathrm{Tc} \mathrm{ml}{ }^{-1}$. The recA mutation of the transductants was checked by a UVsensitivity test (Maniatis et al., 1982). Tc-sensitive clones of the $\operatorname{rec} A$ deletion derivatives were selected on LB agar plates containing chlortetracycline hydrochloride, fusaric acid and $\mathrm{ZnCl}_{2}$ (Maloy \& Nunn, 1981 ; Bochner et al., 1980). Selection of rec $A^{+}$revertants was avoided by confirming $\operatorname{rec} A$ mutations of $E$. coli hosts for cosmid cloning just before their use by the UV-sensitivity test described above.
Appropriate UV-sensitive clones were used for cosmid cloning, or stored at $-80{ }^{\circ} \mathrm{C}$ in the presence of $15 \%(\mathrm{v} / \mathrm{v})$ glycerol or $7 \%(\mathrm{v} / \mathrm{v})$ dimethylsulphoxide until used. LB (Maniatis et al., 1982) was routinely used to grow bacterial cultures. LB agar plates contained $1.2 \%(\mathrm{w} / \mathrm{v})$ Bactoagar in LB. Handling of bacteria, manipulation of DNA for molecular cloning, and restriction mapping of cosmids and deleted cosmids were carried out as described by Maniatis et al. (1982). DNA sequencing was done by the chain-termination method (Sanger et al., 1977).

Cosmid libraries. We constructed cosmid libraries for mouse $\mathrm{L}$ cell genomic DNA with cosmid vectors pJB8 (Ish-Horowicz \& Burke, 1981), pDC1 (Ishiura et al., 1989a), pDC2 (Ishiura et al., 1989a) and pDCג1 (Ishiura et al., 1989a). We also constructed cosmid libraries for CHO cell genomic DNA and Haemophilus influenzae genomic DNA with the $\mathrm{pDC} 1$ vector and the pDC104 vector (Ishiura et al., 1989a), respectively. Vector $\mathrm{pDC} \lambda 1$ is a derivative of $\mathrm{pDCl}$, which carries a 2.8 $\mathrm{kb}$ Nhe I-Xhol segment containing ori of phage $\lambda$ derived from loric (Little \& Cross, 1985) in place of that of pBR327 (M. Ishiura and coworkers, unpublished data). An equimolar mixture of the left and right vector arms of almost equal length was prepared from the vector DNA, simply by treating the DNA sequentially with three enzymes, $P v u I I$ (or Clal in the case of pDC $\lambda 1$ ), calf intestinal alkaline phosphatase, and $B a m H I$ (or $B g / I I$ in the case of pDC2), as described previously (Ishiura et al., 1989a). An equimolar mixture of the left and right arms of pJB8 was prepared as described by Ish-Horowicz \& Burke (1981). Insert DNAs were prepared by the partial digestion of various genomic DNAs with Sau3AI and subsequent size-selection of the partial digests on a sucrose density gradient, as described previously (Ishiura et al., $1989 a$ ). The equimolar mixture of the vector arms, which carried a single cos oriented from left to right, was ligated with insert DNA of $40-50 \mathrm{~kb}$. The ligated recombinant molecules were packaged into $\lambda$ phage particles by an in vitro packaging reaction using an Amersham packaging kit. Among the ligation products, only recombinant molecules which have a structure of 'left arm-insert-right arm' can be converted into transducing $\lambda$ phage particles. Various $E$. coli strains were infected with $\lambda$ phage particles of each cosmid library. To prevent double transduction of cosmids, about 2000 colony-forming units of packaged cosmids were infected into more than $2 \times 10^{8}$ host bacterial cells. The transductants carrying cosmids were selected on LB agar plates containing $30 \mu \mathrm{g} \mathrm{Ap} \mathrm{ml}^{-1}$ or $10 \mu \mathrm{g} \mathrm{Tc} \mathrm{ml}^{-1}$. To avoid the formation of double colonies, cells were plated at low density (about 50 150 drug-resistant colonies per $90 \mathrm{~cm}$ plate). We also constructed a

Table 1. E. coli strains

\begin{tabular}{|c|c|c|}
\hline Strain* & Relevant genotype & Source (reference) \\
\hline HB101 & recA13 hsd $S 20$ & Our stock (Boyer \& Roulland-Dussoix, 1969) \\
\hline ED8767 & recA56 hsdS3 & A. R. Flavell, B. J. Bachmann (Murray et al., 1977) \\
\hline DH1 & recAl hsdRI7 & D. Hanahan (Hanahan, 1983) \\
\hline RRI & As $\mathrm{HB} 101$ but rec $^{+}$ & Bethesda Research Laboratories (Maniatis et al., 1982) \\
\hline ED8654 & As ED8767 but rec $h s d R 514 \operatorname{trpR} 55$ & B. J. Bachmann (Borck et al., 1976) \\
\hline MM294 & As DHl but $\mathrm{rec}^{+} \mathrm{gyr} A^{+}$ & B. J. Bachmann (Meselson \& Yuan, 1968) \\
\hline $\mathrm{C} 600 \mathrm{r}^{-} \mathrm{m}^{-}$ & $\mathrm{rec}^{+} h s d R$ hsdM & K. Matsubara \\
\hline MI1 & As $\mathrm{C} 600$ but $\operatorname{rec} A(\Lambda)$ & This study \\
\hline MI2 & As ED8654 but $\Delta(r e c A-s r l R) 306:: \operatorname{Tn} 10$ & This study \\
\hline SK 1592 & $s b c B 15$ hsdR4 & J. D. Diller (Kushner, 1978) \\
\hline CES200 & recB2I recC22 sbcB15 hsdR & C. Shurvinton \& F. Stahl (Nader et al., 1985) \\
\hline CES201 & As for CES200 but $\Delta(r e c A-s r l R) 306:: \operatorname{Tn} 10$ & C. Shurvinton \& F. Stahl \\
\hline DB1161 & recB21 recC22 sbcB15 recA56 hsdR hsdM & A. R. Wyman \& D. Botstein \\
\hline $\mathrm{JC} 10289$ & $\Delta(r e c A-s r l R) 306:: \operatorname{Tn} 10$ & B. J. Bachmann (Csonka \& Clark, 1979) \\
\hline
\end{tabular}

*All strains except JC10289 were deficient in the major restriction activity. CES200, CES201, and DB1161 are derivatives of AB1157 (rac). Thus, these strains are also rac. 


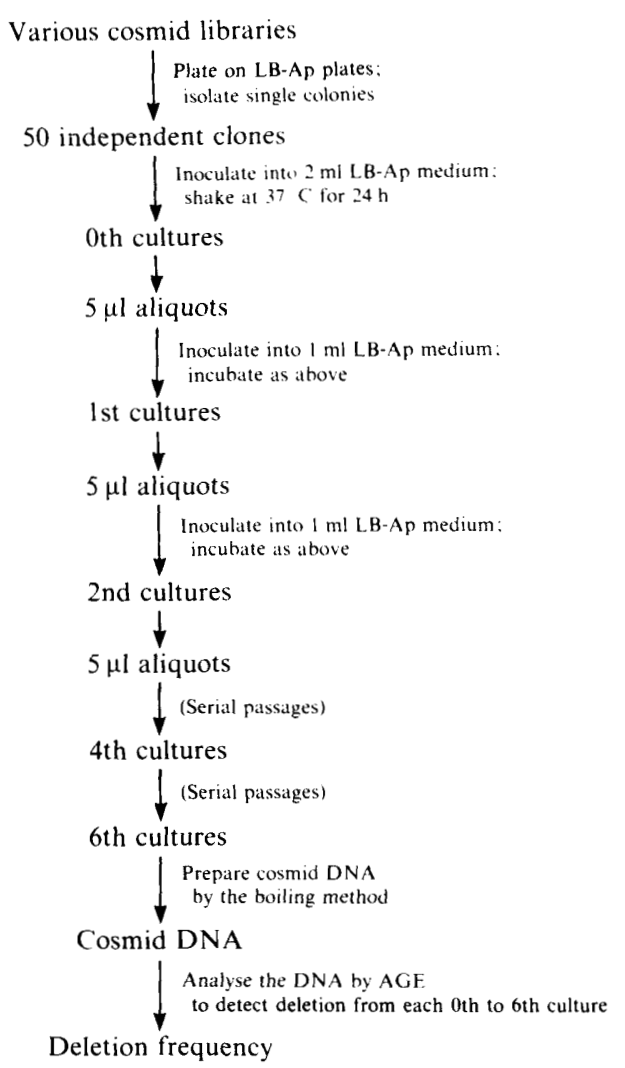

Fig. 1. Scheme for deletion assay. See text for details.

library for L cell genomic DNA with the $\mathrm{pDCl}$ vector, and isolated three cosmids, cMB7, cMB15 and cMB30, each of which carried single BamHI fragments of 40-50 kb (Ishiura et al., 1989a). Essentially all cosmids constructed by our procedures (Ishiura et al., 1989a) contained insert DNA and one copy of vector.

Assay of deletion in cosmid DNAs (see Fig. I). Fifty independent Apresistant colonies (or Tc-resistant colonies for cosmids constructed with pDC2) were isolated from each cosmid library, separately inoculated into $2 \mathrm{ml} \mathrm{LB}$-Ap medium (or LB-Tc medium) in small test tubes, and then incubated at $37^{\circ} \mathrm{C}$ for $24 \mathrm{~h}$ with shaking at 175 r.p.m. These cultures are designated as ' 0 th cultures'. Then $5 \mu \mathrm{l}$ volumes of each 0 th culture were inoculated into $1 \mathrm{ml}$ fresh LB-Ap medium (or LBTc medium) and cultured at $37^{\circ} \mathrm{C}$ for $24 \mathrm{~h}$ as described above to obtain the 'Ist cultures'. Serial cultures were continued to the '6th cultures". For detection of possible deletions in the cosmids, cosmid DNAs were prepared by the boiling method (Holmes \& Quigley, 1981) from $1 \mathrm{ml}$ samples of each 0th to 6th culture and analysed by agarose gel electrophoresis (AGE) in $0.8 \%$ agarose gel in $40 \mathrm{~mm}$-Tris/acetate buffer (pH 8.0) containing 2 mM-EDTA (Maniatis et al., 1982).

\section{Results}

\section{recA-independent deletion in cosmids}

E. coli rec $A$ strains HB101, ED8767 and DH1 have been used as standard hosts and $\mathrm{pJB} 8$ as the standard vector for cosmid cloning. When we constructed cosmids using the pJB8 vector and insert DNAs prepared from $L$ cell genomic DNA and propagated the cosmids in these strains, only $0-4 \%$ clones showed deletion at the 0 th culture, but the frequency increased greatly during serial cultures (Table 2): $34-50 \%$ clones showed deletions at the 2 nd culture, $52-92 \%$ at the 4 th culture, and $54-100 \%$ at the 6th culture. Although more than half the clones showed deletions after extensive serial cultures, clearly some clones did not show any deletion even after prolonged serial cultures. This finding suggests that the probability of deletions depends on the insert DNA. The frequencies of deletions in three $\operatorname{rec} A$ strains, HB101, ED8767 and DH1, were essentially the same.

Fig. 2(a) shows AGE patterns of cosmids constructed with pJB8 and propagated in HB101. We could not detect any deletion in any of the 16 clones at the 0 th culture, but detected deletions in all clones except no. 15 at the 6 th culture. The pattern of deletion bands differed from clone to clone (see also Fig. 2b). Fig. 2(b) shows the changes in deletion band patterns during cultures of four typical clones propagated in three strains, HB101, ED8767 and DH1. Initially deletion bands were weak, if detected at all, but they became clearly evident at the 2 nd or 4th culture and then the patterns did not change during further culture. The deletion band pattern seemed to be specific for each clone.

We determined whether the $\operatorname{rec} A$ mutation of the $E$. coli host was maintained stably during the experiments described above by a UV-sensitivity test (Maniatis et al., 1982). HB101 host cells carrying cosmid DNAs with deletions at the 6th culture were found to be as sensitive to UV-irradiation as those carrying the intact cosmid DNA at the 0th culture, suggesting that the recA mutation did not revert to the $\operatorname{Rec} \mathrm{A}^{+}$phenotype during liquid culture.

To determine whether the rec $A$ gene is involved in the deletions in cosmids, we examined the deletions in cosmids during propagation in three $\mathrm{rec}^{+}$strains, RR 1 (as HB101 but rec ${ }^{+}$, ED8654 (as ED8767 but $\mathrm{rec}^{+}$ hsdR514 trpR55), and MM294 (as DH1 but rec ${ }^{+} \operatorname{grAA}^{+}$). With the exception of MM294, the frequencies of deletions in these $\mathrm{rec}^{+}$strains were almost the same as or slightly higher than those obtained with the corresponding $\operatorname{rec} A$ strains (see below). The frequencies observed with MM294 were rather lower than those with the corresponding $\operatorname{rec} A$ strain $\mathrm{DH} 1$.

Effect of a rec $A$ deletion mutation in the $E$. coli host on the deletions in cosmids

The data described above showed that deletions in cosmids occurred irrespective of the rec $A$ mutations of the $E$. coli hosts. However, we could not completely rule 
Table 2. Deletion of recombinant cosmid DNA during propagation in E. coli hosts grown in liquid cultures

Fifty independent cosmids, propagated in the indicated E. coli strains, from each cosmid library (constructed using the vectors and insert DNAs described below) were cultured, with daily serial passages, in LB-Ap medium at $37^{\circ} \mathrm{C}$ with shaking. Cosmid DNA was prepared from the cells at the 0 th to 6 th culture and analysed by AGE to detect any deletion in the DNA. All strains were deficient in the major restriction activity.

\begin{tabular}{|c|c|c|c|c|c|c|c|}
\hline \multirow[b]{3}{*}{ Host } & \multirow[b]{3}{*}{ Relevant genotype } & \multirow[b]{3}{*}{ Vector } & \multirow{3}{*}{$\begin{array}{l}\text { Insert } \\
\text { DNA }\end{array}$} & \multicolumn{4}{|c|}{$\begin{array}{c}\text { Deletion frequency } \\
\text { (no. of deleted clones per } 50 \text { clones) }\end{array}$} \\
\hline & & & & \multicolumn{4}{|c|}{ Culture: } \\
\hline & & & & 0 th & 2nd & 4th & 6 th \\
\hline HB101 & $\operatorname{rec} A 13$ & pJB8 & $L$ cell & 2 & 20 & 31 & 36 \\
\hline HB 101 & $\operatorname{rec} A 13$ & pDC1 & L cell & 0 & 16 & 47 & 49 \\
\hline HB101 & $\operatorname{rec} A 13$ & $\mathrm{pDC} \lambda \mathrm{l}$ & $\mathrm{L}$ cell & 0 & 10 & 50 & ND \\
\hline ED8767 & $\operatorname{rec} A 56$ & pJB8 & $L$ cell & 0 & 17 & 26 & 27 \\
\hline ED8767 & $\operatorname{rec} A 56$ & $\mathrm{pDCl}$ & $\mathrm{L}$ cell & 0 & 23 & 33 & 37 \\
\hline DH1 & recAl & pJB8 & L cell & 2 & 25 & 46 & 50 \\
\hline RR1 & $\mathrm{rec}^{+}$ & pJB8 & $\mathrm{L}$ cell & 0 & 24 & 31 & 41 \\
\hline ED8654 & $\mathrm{rec}^{+}$ & pJB8 & $\mathrm{L}$ cell & 2 & 35 & 41 & 45 \\
\hline $\mathrm{C} 600 \mathrm{r}^{-} \mathrm{m}^{-}$ & $\mathrm{rec}^{+}$ & pJB8 & $\mathrm{L}$ cell & 0 & 45 & 45 & 46 \\
\hline MM294 & $\mathrm{rec}^{+}$ & pJB8 & $\mathrm{L}$ cell & 0 & 10 & 13 & 23 \\
\hline MII & $\operatorname{rec} A(\Delta)$ & $\mathrm{pJB} 8$ & $\mathrm{~L}$ cell & 3 & 27 & 41 & 46 \\
\hline MII & $\operatorname{rec} A(\Delta)$ & $\mathrm{pDCl}$ & $\mathrm{L}$ cell & 1 & 14 & 36 & 45 \\
\hline MI2 & $\operatorname{rec} A(\Delta)$ & $\mathrm{pJB} 8$ & L cell & 0 & 37 & 48 & 50 \\
\hline MI2 & $\operatorname{rec} A(\Delta)$ & $\mathrm{pDCl}$ & $\mathrm{L}$ cell & 1 & 41 & 50 & ND \\
\hline $\mathrm{HB} 10 \mathrm{l}$ & recA13 & pDCl & H. influenzae & 1 & 29 & 47 & 47 \\
\hline HB101 & $\operatorname{recA} 13$ & pDC104 & CHO cell & 1 & 11 & 42 & 47 \\
\hline SK 1592 & $s b c B 15$ & $\mathrm{pDCl}$ & $\mathrm{L}$ cell & 3 & 32 & 48 & 49 \\
\hline CES200 & $\operatorname{rec} B 21 \operatorname{rec} C 22 \operatorname{sbc} B 15$ & $\mathrm{pDCl}$ & $\mathrm{L}$ cell & 3 & 20 & 33 & 37 \\
\hline CES201 & $\operatorname{rec} B 21 \operatorname{rec} C 22 \operatorname{sbcB} 15 \operatorname{rec} A(\Delta)$ & $\mathrm{pDCl}$ & L cell & $0^{*}$ & $20^{*}$ & ND & ND \\
\hline DB1161 & recB21 recC $22 \operatorname{sbcB} 15$ rec $A 56$ & $\mathrm{pDCl}$ & $\mathrm{L}$ cell & 27 & 44 & 47 & 47 \\
\hline
\end{tabular}

ND, Not determined.

*Numbers of clones with deletions per 20 clones. Only a very few transductant colonies of CES201 could be obtained.

out the possibility that the observed deletion was due to leaky function of the mutated $\operatorname{rec} A$ genes of the $E$. coli hosts. To examine this possibility, we constructed two $E$. coli strains, MI1 and MI2, which carried a $\operatorname{rec} A$ deletion mutation, and used them as hosts for propagation of cosmids. We also used the two $\mathrm{rec}^{+}$parental strains, C600 and ED8654, as control hosts (Table 2, Fig. 2c).

Neither MI1 nor MI2 prevented deletions, and the deletion frequencies obtained with the two strains did not differ much from those obtained with the three $\operatorname{rec} A$ strains, HB101, ED8767 and DH1 $(50-70 \%$ at the 2 nd culture, $82-96 \%$ at the 4 th, and $92-100 \%$ at the 6 th) (Table 2). The deletion frequencies obtained with MI2 were also almost the same as those obtained with its $\mathrm{rec}^{+}$ parental strain ED8654. The deletion frequencies obtained with MI1 were slightly lower than those obtained with its $\mathrm{rec}^{+}$parental strain $\mathrm{C600}$. The deletion band patterns shown in Fig. 2(c) were also characteristic of each clone.

\section{Effects of vectors on the deletion}

There is a possibility that the vector DNA and ori of the vector might affect the deletion in cosmids. To test this possibility, we examined the effects of three other

Fig. 2. AGE patterns of cosmid DNAs propagated in E. coli hosts. Cosmids were constructed using the vectors specified and inserts prepared from L cell genomic DNA [except in $(d)$ ], and were propagated in $E$. coli hosts as indicated in parentheses. After serial cultures as indicated, cosmid DNA was analysed by AGE. (a) Sixteen cosmids constructed with the pJB8 vector and propagated in HB101. (b) Changes during serial culture in deletion bands of the typical cosmids propagated in HB101, ED8767 and DH1. Results for 16 independent clones are shown. Results for the 0th, 2nd, 4th, and 6th cultures of each clone are shown from left to right. (c) Deletion band patterns of the cosmids constructed with the $\mathrm{pJB} 8$ vector and propagated in two $\operatorname{rec} A(\Delta)$ strains, MI1 and MI2, and their parental rec ${ }^{+}$ strains C600 and ED8654. Results for 16 independent clones are shown. Results for the 0th and 2nd cultures of each clone are shown from left to right. (d) Deletion band patterns of the cosmids derived from the cosmid libraries for the genomic DNAs of CHO cells and Haemophilus influenzae, which were constructed with the pDC104 vector and the pDC1 vector, respectively. Results for the 0 th and 2 nd cultures of eight independent clones are shown from left to right. 
(a)

pJB8(HB101)

0th culture

6 th culture

$\begin{array}{llllllllllllllll}1 & 2 & 3 & 4 & 5 & 6 & 7 & 8 & 9 & 10 & 11 & 12 & 13 & 14 & 15 & 16\end{array}$

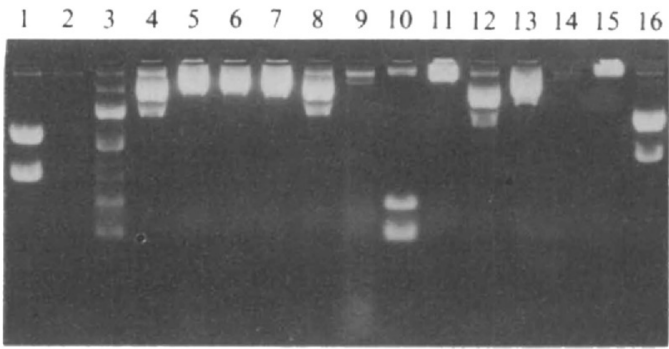

(b)

pJB8(HB|01)
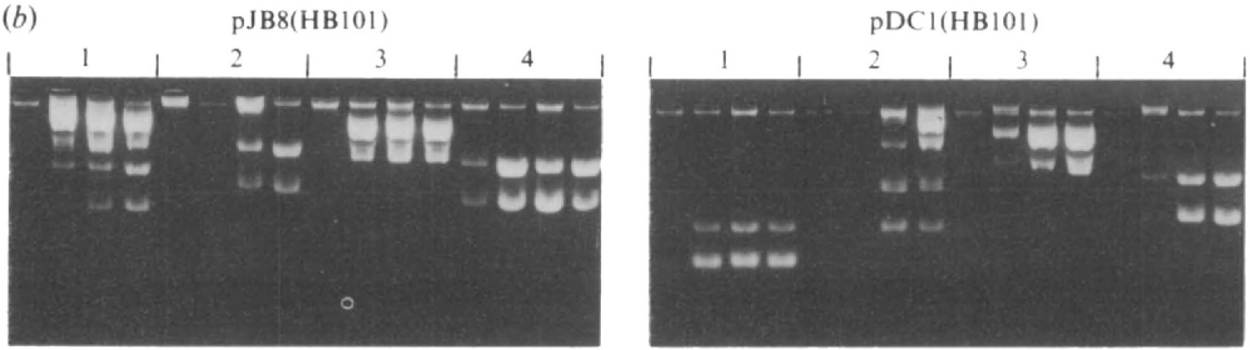

pJB8(DH1)

pJB8(ED8767)
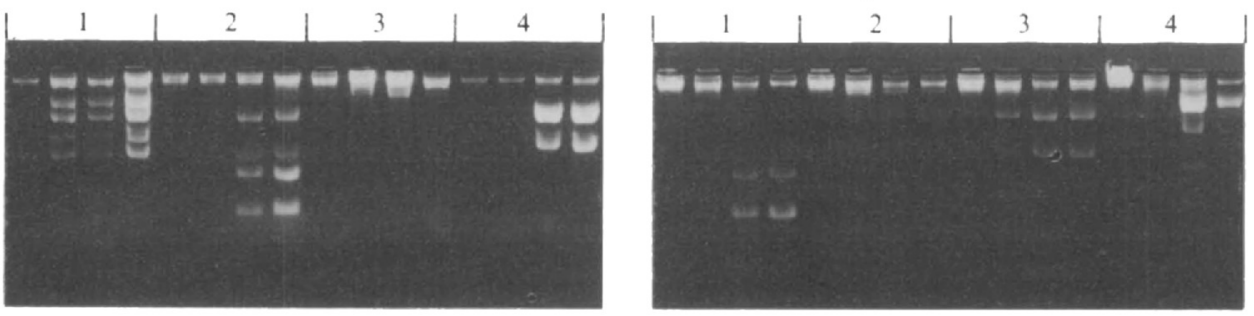

(c)

pJB8(C600)

pJB8(ED8654)

$\mathrm{rec}^{+} \quad \operatorname{rec} A(\Delta)$

$\mathrm{rec}^{+} \quad \operatorname{rec} A(\Delta)$
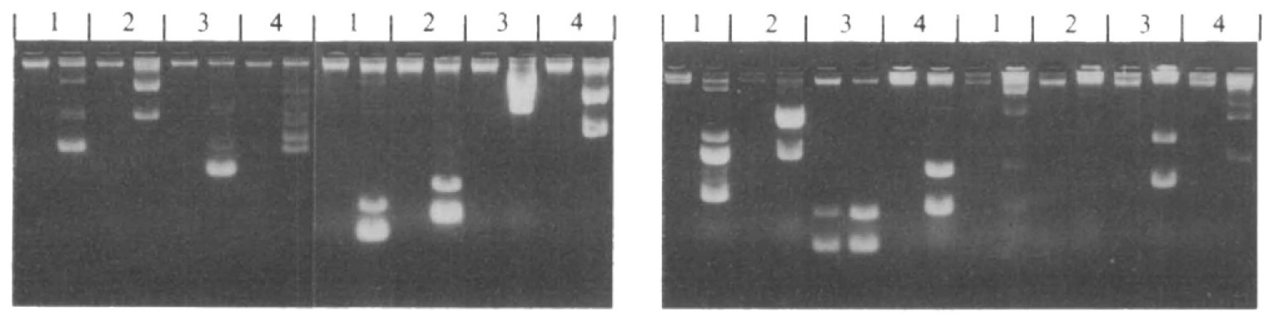

(d)

$\mathrm{pDC} 1(\mathrm{HB} 101)$

$H$. influenzae

pDC104(HB101)

$\mathrm{CHO}$
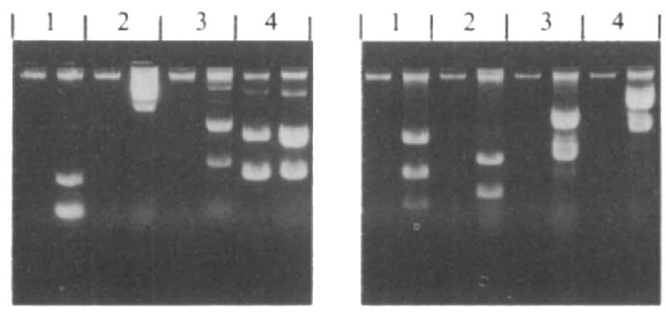

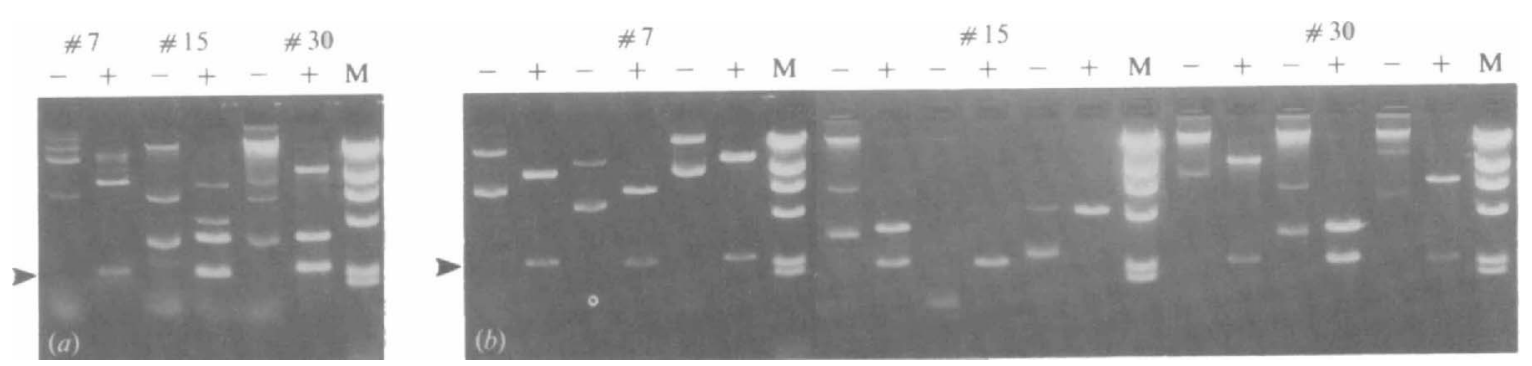

Fig. 3. AGE analysis of cosmids with single BamHI inserts after extensive amplification in $E$. coli hosts. Three cosmids, cMB7, cMB15, and cMB30, which carried single BamHI inserts, were propagated in HB101 until the 6th culture. \# 7, \# 15, and \# 30, DNAs of cMB7, cMB15, and cMB30 with deletions; + and -, with and without BamHI digestion, respectively; $\mathrm{M}$, size markers ( $\lambda$ HindIII digests); the arrowheads indicate the position of the vector band. (a) AGE patterns of cosmid DNAs with deletions prepared from the 6th culture. (b) Analysis of cosmid DNAs with deletions by retransformation. Cosmid DNAs with deletions obtained from the 6th cultures as described in $(a)$ were reintroduced into $\mathrm{HB} 101$; three independent transformants of each cosmid were isolated and their cosmid-derived DNAs were analysed, directly or after BamHI digestion, by AGE.

vectors, $\mathrm{pDC} 1, \mathrm{pDC} 104$ and $\mathrm{pDC} \lambda 1$. $\mathrm{pDC} \lambda 1$ is a derivative of loric that carries ori of phage $\lambda$ (Little \& Cross, 1985). The deletion frequencies with these three vectors did not differ much from those with the pJB8 vector (Table 2). Thus, at least with these vectors, the vector per se did not seem to have a large effect on the deletion frequency. The level of resistance to $A p$ is proportional to the number of copies of bla. Generally, the copy number of pMB-derived plasmids in $E$. coli host cells is inversely related to plasmid size. Therefore, although the concentration of Ap in the culture media used here was relatively low $\left(30 \mu \mathrm{g} \mathrm{ml}^{-1}\right)$, it is possible that the formation of deletions in cosmid DNA may provide a growth advantage for host cells carrying cosmids in medium containing Ap. However, the deletions did not seem to be due to bla carried on these four vectors, $\mathrm{pJB} 8, \mathrm{pDCl}, \mathrm{pDC} 104$ and $\mathrm{pDC} \lambda 1$, because use of the $\mathrm{pDC} 2$ vector, a derivative of $\mathrm{pDC} 1$ that carries the Tc-resistance gene of pBR322 in place of bla, did not prevent deletions (data not shown).

\section{Effects of the sources of insert DNAs on the deletion}

To determine whether cosmid deletion events are general phenomena that occur in any DNA, we used genomic DNAs of CHO cells and Haemophilus influenzae as inserts. The deletion frequencies of the cosmids with these inserts did not differ much from those of cosmids with $\mathrm{L}$ cell genomic DNA (Table 2). Fig. 2(d) shows the changes in deletion band patterns during cultures of eight clones. Initially deletion bands were not detected in most clones, but they became evident at the 2 nd cultures or the 4 th cultures and then the patterns did not change during further culture.
Effects of a recB recC $s b c B$ host and related hosts on the deletion

In both $\lambda$ and plasmid cloning, a $\operatorname{rec} B s b c B$ host and a $\operatorname{rec} B \operatorname{rec} C \operatorname{sbc} B$ host have been reported to prevent the deletions of some kinds of recombinant DNAs that were not stably maintained in usual $\mathrm{rec}^{+}$or recA hosts (Collins et al., 1982; Leach \& Stahl, 1983; Wyman et al., 1985; Nader et al., 1985). Therefore, we examined the effects of such $E$. coli hosts on the cosmid deletion events. However, the deletion frequency was not reduced by an $s b c B$ host, a $r e c B$ rec $C s b c B$ host, or a $r e c B$ rec $C s b c B$ rec $A$ host (Table 2).

\section{Approximate location of the deletions}

We determined the approximate location of deletions in cosmids propagated in $\mathrm{HB} 101$, using three test cosmids, cMB7, cMB15 and cMB30, each of which carried single BamHI inserts of $40-50 \mathrm{~kb}$. Fig. 3(a) shows the AGE patterns of DNAs of these cosmids at the 6th culture. After BamHI digestion, a clear vector band was seen, indicating that the vector portions were intact in most cosmid molecules with deletions and that the deletions were mainly in the insert DNAs.

For confirmation of these results, we reintroduced each DNA of these cosmids with deletions into HB101 cells, isolated the three independent transformants for the three cosmids, and analysed the deleted cosmid DNAs by AGE. Fig. 3(b) shows that in all nine clones except clone no. 15-3 each deletion band was composed of intact vector DNA and deleted insert DNA. In clone no. 15-3, the deletion end-points occurred in both the vector and the insert, eliminating one of the BamHI junction sites between the vector and the insert. 


\section{Nucleotide sequences surrounding deletion junctions}

We analysed the nucleotide sequences that surround deletion junctions in the cMB15 test cosmid. Restriction mapping experiments showed the existence of deletion hot-spots in the cMB15 DNA propagated in $\mathrm{HBl} 101$ $(\mathrm{rec} A)$ and RRl $\left(\mathrm{rec}^{+}\right)$. Some deleted cosmids had deletion junctions at almost identical sites irrespective of whether the cosmid had been propagated in HB101 or RR1 (Fig. 4a). All six deletions examined had occurred in the insert portions between two short complete direct repeats of about 4-10 bp which were separated from each other by about $40 \mathrm{~kb}$ in one direction and about $4 \mathrm{~kb}$ in the other direction, in both HBl01 and RR1 (Fig. 4). Two deleted cosmids propagated in $\mathrm{HB} 101, \mathrm{H} 33$ and H35 had an identical deletion junction (D1). Another two deleted cosmids propagated in RR1, R9 and R23 also had an identical deletion junction (D2). The right break-point (DR3) of one deletion junction (D3) observed in the deleted cosmid R13 that had been propagated in RR1 was found within that (DR2) of the deletion junction (D2) observed in R9 and R23. Although R29 had been propagated in RR1, it had the same deletion junction (D1) as that observed in H33 and H35.

Various sequence motifs were observed around the deletion junctions (Fig. $4 b$ ). They were sequences related to the Chi sequence (5'-GCTGGTGG-3' or its complementary sequence $5^{\prime}$-CCACCAGC- $3^{\prime}$ ), and sequences related to the specific 8 bp sequence $5^{\prime}$-GAGGC ${ }_{\mathrm{GT}}^{\mathrm{TG}}$ (or

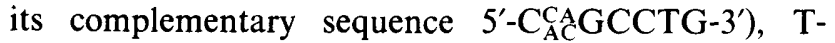
stretches (or A-stretches), C-stretches (or G-stretches). One deletion junction (D1) contained the Chi-related sequence. The tandem repeats of $5^{\prime}-\mathrm{CCT}-3^{\prime}$ (or its complementary sequence $5^{\prime}-\mathrm{AGG}-3^{\prime}$ ) and $5^{\prime}-\mathrm{CCA}-3^{\prime}$ (or its complementary sequence $5^{\prime}$-TGG- $3^{\prime}$ ) were observed in and near all six deletion junctions examined here (Fig. $4 c$ ). Inverted repeat sequences and symmetric sequences were also observed around deletion junctions. Among six break-points of the deletion junctions examined, three (DL2, DR2 and DR3) were found within such symmetric sequences, and two (DL3 and DR1) overlapped with such inverted repeat sequences. Tandem repeat sequences of more than $10 \mathrm{bp}$ were also found in the flanking regions of the deletion junctions. One deletion junction (D1) was coincident with one such tandem repeat sequence.

\section{Discussion}

By analysing the DNAs of more than 1000 independent cosmids propagated in various $E$. coli hosts, we demonstrated that most cosmids were not stably maintained, but suffered deletions, in $E$. coli hosts, including recA strains, such as HB101, ED8767 and DH1, which have generally been used as hosts for cosmid cloning. As observed previously (Ishiura et al., 1989 b), recA mutations in $E$. coli hosts do not reduce the frequency of cosmid deletion events. This was not due to back mutations of the host rec $A$ gene to the $\operatorname{Rec}^{+}$phenotype (Chia et al., 1982) since the deletion occurred similarly in $E$. coli hosts carrying a rec $A$ deletion mutation. Therefore, most of the cosmid deletion events described here were $\operatorname{rec} A$ independent.

The deletion frequency and deletion band pattern of cosmid DNA differed from clone to clone. Furthermore, the deletion in cosmids occurred mainly in the insert DNA. These findings suggest that a specific sequence(s) or structure(s) that is often present in insert DNA was responsible for the deletions (see below). This specific sequence(s) or structure(s) of genomic DNA is present not only in mammalian cells but also in micro-organisms such as Haemophilus influenzae, because similar deletions in cosmids occurred irrespective of whether mammalian or $H$. influenzae genomic DNA was used as a source of the insert DNA.

Nucleotide sequence analysis demonstrated that all deletions in test cosmid cMB15 examined here occurred between two short complete direct repeats. Even deletions in the cosmid observed during propagation in a $\mathrm{rec}^{+}$host occurred between two short direct repeats of about $4-10 \mathrm{bp}$. Homologous recombination mediated by the rec $A$ recombination system usually involves larger homologous sequences (Shen \& Huang, 1986). Furthermore, some deletions occurred at the same sites irrespective of whether the test cosmid was propagated in a $r e c A$ host or a $\mathrm{rec}^{+}$host. These findings also suggest the $\operatorname{rec} A$ independence of the cosmid deletion events. Similar recA-independent deletion events involving a short direct repeat have been also reported in plasmids (Albertini et al., 1982; Jones et al., 1982). However, such deletions occurred 25 -fold more frequently in a rec ${ }^{+}$host than in a rec $A$ host (Albertini et al., 1982). We observed various sequence motifs, including Chi-related sequences, sequences related to the $8 \mathrm{bp}$ sequence 5'GAGGC TGG (or 5'-C CACCCTG-3'), T-stretches (or A-stretches), C-stretches (or G-stretches), and the tandem repeats of 5'-CCT-3' (or 5'-AGG-3') and 5'-CCA-3' (or 5'-TGG-3'), around the deletion junctions (Fig. $4 b$ ). Inverted repeat sequences and symmetric sequences were also observed around deletion junctions. Recently, we analysed the deletion junctions observed in human test cosmid cHEF2-12, which carries the human elongation factor 2 gene (Ishiura et al., 1989b) that had been propagated in a rec $A$ E. coli host and found that deletions also occurred between two short direct repeats of 6-25 bp (M. Ishiura and co-workers, unpublished data). The 
(a)

cMB15

H33, H35

R29

R9, R23

R13
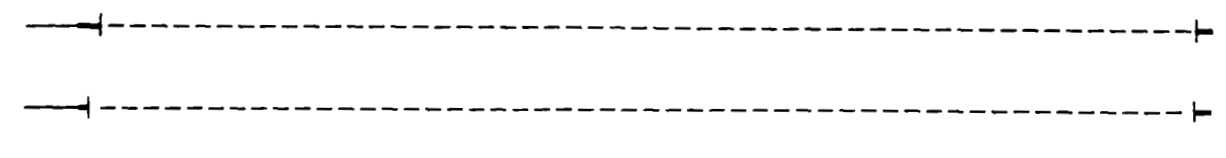

(b)

40

50

60)

70

80

90

100

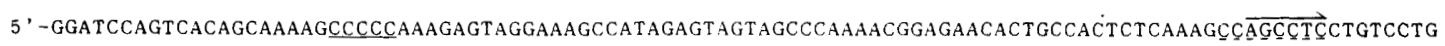
ATCTGACCTCATCTGACCTACCT $\widetilde{G G G G C T C C T C T A C A T C A G T T T G T A C T G C T G C C C T T C C A C A C A G G T C C C C A C C A C A C A T G A C C T A}$ TAGCATCCCTGC CCCCAGCCCTGTGTGAGTTGTCCTTGCCACCGTCTCCTCTTCCAGATCCAATCATGTTCCTTTTGGCATGACGATT TTCCCAGCAGCCCTGGCTACACCA

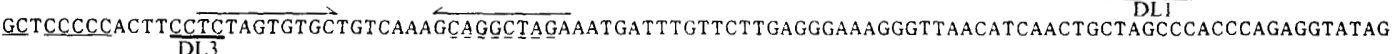
TTGGTATATTCCAAGCTGCTAATTTTTATTTTATTTTATTTTTI GCTTAGGGAGATGTAAAGTCAGCCCCPCCTCCTAAGGTCTCCAGGGCAAAGTGA GGTATGATTTTACCAATCTTCACTCTGGAGCCTATGAGTTTAGTAAGTATACTCACAGGGCTGTGGGGATTATGGGCAGGAGTGTGGGTGGCCTTAACAC AGCCACACTAGAAGTCTACCAGCTTGGGCGAGGaGTTCCCAATGGACCAATAGATGAGAATCCTTCTCCCTAGACCT TTCCAGCCIATGTACTTCTAGCC

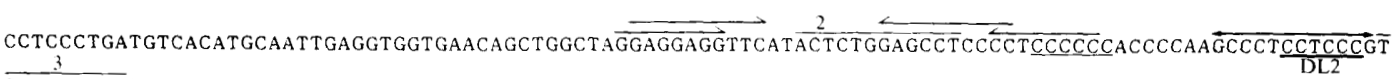
$\frac{3}{\text { GAGAAGCATCACAGTCAACAGC }-10} \mathrm{~kb}$ CCCAGTGACATTCATTAAATGGTCAGCCACAAATATATTTGGTCTTATGAGCTCATTGATGATAGATT AAAGAGGATCAACCTCTATT GAACATGTGTTG

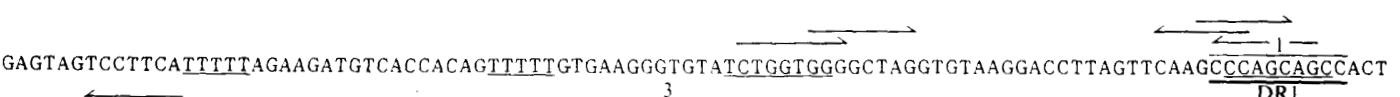
TGAGAAGTTGGGCATCCTTGCATTGATATT TATAAACTGCTTCTGAGAAAGCAGAGACAGGAGGACCGACCCCTGGGGCTTTGGGGGGGGGGCAGCCAGT

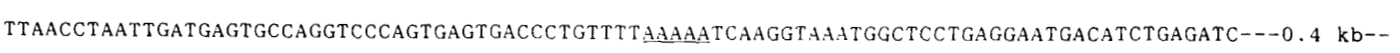

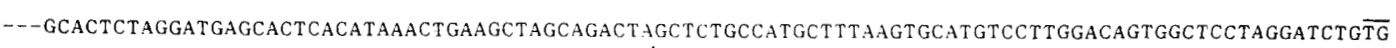

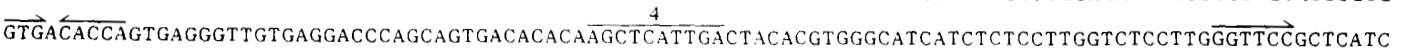
AGGGACCAGAATTCCTTGAGACGTCACGAAAGGATTCTGAAGTCAGTGAGAGATGTGATCCCACCCACCCTCCCACCCCGCCCCCAGTGAaGCCCCTA GATACTCACCGTCTGCTTTGGAGCCTCGa-3.

$\frac{\mathrm{DR} 2}{\mathrm{DR} 3}$

(c)

\section{H33, H35, R29:}

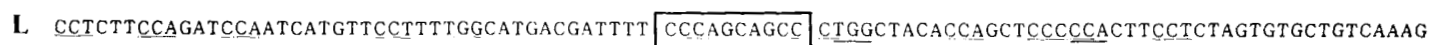

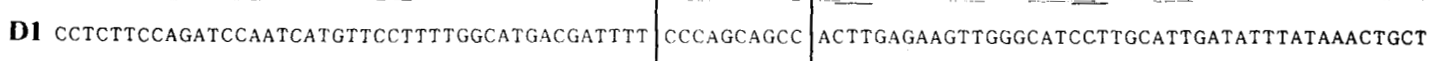

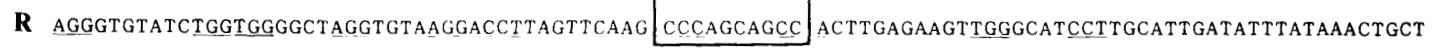

\section{R9, R23:}

L CATACTCTGGAGCCTCCCCTCCCCCCACCCCAAGCCCT CCTCCC GTGAGAAAGCATCACAGTCAACAGC

D2 CATACTCTGGaGCCTCCCCTCCCCCCACCCCAAGCCCT CCTCCC ACCCCGCCCCCAGTGAAAGCCCCTAGATACTCACCGTCTGCTTTGGAGCCTCG

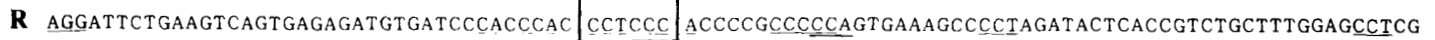

\section{R13:}

L GATTTTCCCAGCAGCCCTGGCTACACCAGCTCCCCCACTT CCTC TAGTGTGCTGTCAAAGCAGGCTAGAAATGATTTGTTCTTGAGGGAAGGGTTA

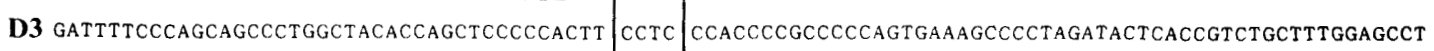

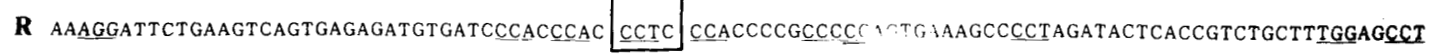


sequence motifs and DNA structures described here were also found around the deletion junctions observed in cHEF2-12 (M. Ishiura and co-workers, unpublished data).

Previously, we showed that $r e c B$ rec $C s b c B$ recJ hosts prevent the cosmid deletion events which occur in recA hosts, using six test cosmids including $\mathrm{cMB} 15$ (Ishiura et $a l ., 1989 b$ ). Thus, it appears that a $\operatorname{rec} B$ recC $s b c B$ recJ quadruple combination of host mutations prevents deletions in cosmids which occur between short direct repeats. It has been reported that most of various rec $A$ independent deletion events observed in $E$. coli involve short direct repeat sequences or short inverted repeat sequences (for a review, see Allgood \& Silhavy, 1988), and two models have been proposed to explain such deletion events (Franklin, 1971), namely the slipped mispairing and the cutting and rejoining of DNA molecules (homologous recombination). Our present knowledge does not allow us to distinguish between these two mechanisms.

There are reports that a palindromic structure (Collins, 1980), inverted repeat sequences (Lilley, 1981), tandemly repeated sequences (Brutlag et al., 1977), interspersed repeat sequences (Liu \& Lark, 1982), and direct repeat sequences located in the long inverted repeats (Saing et al., 1988), which are cloned in plasmid vectors, are unstable and induce deletions in recombinant plasmids in standard $\operatorname{rec} A$ hosts. These deletions have been reported to be prevented in a $\operatorname{rec} B s b c B$ host (Collins et al., 1982), a recB recC $s b c B$ recF host (Boissy \& Astell, 1985), a recE host (Liu \& Lark, 1982), or a host with a temperature-sensitive mutation in the $g y r A$ gene (Saing et al., 1988). However, we found that an $s b c B$ host, a $r e c B$ rec $C s b c B$ host, and $\operatorname{rec} B$ rec $C s b c B$ rec $A$ hosts did not prevent deletions in cosmids. Our previous experiments suggested that $\operatorname{rec} B$ recC $s b c B$ recF hosts and a $r e c E$ host also did not prevent the deletions (Ishiura et al.,
$1989 b$ ). Preliminary experiments suggest that the temperature-sensitive mutant of the gyrA gene described above (Saing et al., 1988) also does not prevent the deletions (M. Ishiura and co-workers, unpublished data). Plasmidic recombination, namely the multimerization and demultimerization of plasmids and interplasmidic and intraplasmidic recombination, occurs during propagation of plasmids in E. coli (Laban \& Cohen, 1981; Fishel et al., 1981; for a review, see Mahajan, 1988). However, the formation of deletions in cosmids is not likely to be directly related to plasmidic recombination because these two events are differently affected by various mutations involved in recombination in $E$. coli (Ishiura et al., 1989b; Mahajan, 1988). Therefore, the cosmid deletion events of very high frequency, described here, depend mainly on a recombination system(s) other than those reported previously, although the latter recombination systems may be involved secondarily and in our system their involvement may have been masked by the very high frequency of cosmid deletion events.

Currently, it is not easy to analyse the cosmid deletion events of many cosmids using $r e c B$ rec $C s b c B$ recJ strains as host because the efficiencies of transduction of cosmids obtained in such strains are very low (M. Ishiura and co-workers, unpublished data). Therefore, we have attempted to construct a restriction-deficient derivative from a $r e c B$ rec $C s b c B$ recJ strain, JC12186. However, the candidates obtained so far show an efficiency of transduction only ten times higher than that obtained with JC12186 (unpublished data). It remains to be determined whether $\operatorname{rec} B$ rec $C s b c B$ rec $J$ hosts prevent all or most cosmid deletion events that occur in $\operatorname{rec} A$ hosts.

Little \& Cross (1985) suggested that use of a loric vector, which carries or $i$ of phage $\lambda$, in place of the usual pBR322-derived vectors may reduce the frequency of cosmid deletions. Our results presented here and unpublished observations, however, demonstrate that ori of the

\begin{abstract}
Fig. 4. Nucleotide sequences of deletion junctions in the cMB15 test cosmid propagated in a rec $A$ (HB101) or rec ${ }^{+}(\mathrm{RR} 1)$ host. The cMB15 test cosmid DNA was packaged into phage particles in vitro and transduced into HB101 or RR1 cells. Each 20 clones of HB101 or RR1 cells that carried the cosmids were separately cultured, and cosmid DNAs with deletions obtained from the 6th cultures were reintroduced into HB101. Deleted cosmid DNAs obtained from separate cultures were analysed by restriction mapping and nucleotide sequencing. Deleted cosmids, H33 and H35, were obtained from separate HB101 cultures, whereas R9 and R23 were obtained from separate RR1 cultures. (a) Restriction mapping of deletion junctions in cMB15. Ba, BamHI; E, EcoRI; Hp, HpaI; K, KpnI; Sm, SmaI. The thick, thin and dashed lines represent the insert portions, vector portions and deleted portions, respectively. (b) Partial nucleotide sequence of cMB15 which contains the break-points of deletion junctions. The nucleotide sequence is shown from the left end to the right end of test cosmid cMB15 and from the $5^{\prime}$-end to the $3^{\prime}$-end; the left end is defined as the end containing bla and ori (namely, the left arm of pDC1). The left end of the sequence is the left BamHI site of the BamHI insert of cMB15. The left (L) and right (R) sequences of deletion junctions are defined as sequences which contain, respectively, the left (DL1, DL2 and DL3) and right (DR1, DR2 and DR3) break-points of three deletion junctions (D1, D2 and D3) in cMB15. The sequences of the break-points are underlined with bold lines. Sequence motifs, such as sequences related to the Chi sequence (5'-GCTGGTGG-3' or its complementary sequence 5'-CCACCAGC$3^{\prime}$ ), T-stretches (or A-stretches), C-stretches (or G-stretches), are underlined with thinner lines. The sequences related to the $8 \mathrm{bp}$ sequences 5'-GAGGCTGG (or its complementary sequence 5'-C CACGCCTG-3') are shown by the dashed underlines. The arrowed overlines indicate inverted repeat sequences $(\hookrightarrow \leftrightharpoons)$ and symmetric sequences $(\leftrightarrow)$. Direct repeat sequences are shown by the numbered overlines. (c). Nucleotide sequences in the region of deletion junctions. Deletion junctions are boxed. The tandem repeats of $5^{\prime}$-CCT-3' (or its complementary sequence $5^{\prime}-\mathrm{AGG}-3^{\prime}$ ) and $5^{\prime}-\mathrm{CCA}-3^{\prime}$ (or its complementary sequence $5^{\prime}-\mathrm{TGG}-3^{\prime}$ ) are underlined. Other conditions and symbols are the same as described in $(b)$.
\end{abstract}


vectors examined does not seem to have a large effect on the frequency of cosmid deletions. However, there is a possibility that use of a low-copy-number vector may partially solve the deletion problem in cosmid cloning. The cosmid deletion events described here are likely to reflect two processes, the formation of deletions in cosmid DNA and the displacement of original cosmids by deleted forms. The number of copies of intact cosmids seems to be lower than that of the deleted forms. Therefore, the differential rate of replication of cosmids between the intact cosmids and the deleted forms may provide a bias in favour of deletions. In this connection, cosmids may be more stably propagated in $E$. coli host cells if the cosmids are constructed with a low-copynumber vector. To examine this possibility, we have constructed a low-copy-number vector with ori of pSC101 or that of $F^{\prime}$ and examined cosmid cloning with such a vector (unpublished data). However, we have not yet succeeded in increasing the stability of intact cosmids in $E$. coli cells, using a low-copy-number vector.

Fernandez et al. (1986) showed that spontaneous insertion of $E$. coli insertion element IS $l$ into two vectors, pNNL and pTCF, occurred during propagation of the vectors and suggested that use of a vector carrying IS $I$ for cosmid cloning caused a very high frequency of deletions in cosmids in standard $\operatorname{rec} A$ hosts. However, this phenomenon could not account for our observations, because in contrast to the deletions observed in this study, IS 1 -mediated deletions always involve deletions in vector portions starting at the IS 1 segment and eliminate one of the BamHI junction sites between the vector and the insert. In addition, the vectors used here did not carry IS 1 .

We are grateful to B. J. Bachmann, A. R. Flavell, C. Shurvinton and F. Stahl, A. R. Wyman and D. Botstein, J. D. Diller, D. Hanahan, and K. Matsubara for generous gifts of $E$. coli strains. P. F. R. Little for the loric vector, and K. Kita for a Haemophilus cosmid library. We thank T. Koide for his help in DNA sequencing. We also thank S. Hirose and H. Nakashima for critical reading of the manuscript. This work was supported by a grant from the Ishida Foundation and by grants from the Japanese Ministry of Education, Science and Culture (nos 59771747 and 60771985).

\section{References}

Albertini, A. M., Hofer, M., Calos, M. P. \& Miller, J. H. (1982). On the formation of spontaneous deletions: the importance of short sequence homologies in the generation of large deletions. Cell 29, 319-328.

AllgOOD, N. D. \& SilHAVy, T. J. (1988). Illegitimate recombination in bacteria. In Genetic Recombination, pp. 309--330. Edited by R. Kucherlapati \& G. R. Smith. Washington, DC: American Society for Microbiology.

Bochner, B. R., Huang, H.-C., Schieven, G. L. \& Ames, B. N. (1980). Positive selection for loss of tetracycline resistance. Journal of Bacteriology 143, 926-933.
BoIssy, R. \& Astell, C. R. (1985). An Escherichia coli recBC sbcB recF host permits the deletion-resistant propagation of plasmid clones containing the 5'-terminal palindrome of minute virus of mice. Gene 35, $179-185$.

Borck, K., Beggs, J. D., Brammar, W. J., Hopkins, A. S. \& Murray, E. (1976). The construction in vitro of transducing derivatives of phage lambda. Molecular and General Genetics 146, 199-207.

BOyeR, H. W. \& Roulland-Dussoix, D. (1969). A complementation analysis of the restriction and modification of DNA in Escherichia coli. Journal of Molecular Biology 41, 459-472.

Brutlag, D., Fry, K., Nelson, T. \& Hung, P. (1977). Synthesis of hybrid bacterial plasmids containing highly repeated satellite DNA. Cell 10, 509-519.

ChiA, W., SCOtT, M. R. D. \& RigbY, W. J. (1982). The construction of cosmid libraries of eukaryotic DNA using the Homer series of vectors. Nucleic Acids Research 10, 2503-2520.

Collins, J. (1980). The instability of palindromic DNA. Cold Spring Harbor Symposia on Quantitative Biology 45, 409-416.

Collins, J., Volckaert, G. \& Nevers, P. (1982). Precise and nearlyprecise excision of the symmetrical inverted repeats of $\operatorname{Tn} 5$. Common features of rec $A$-independent deletion events in Escherichia coli. Gene 19, 139-146.

Csonka, L. N. \& Clark, A. J. (1979). Deletions generated by the transposon $\mathrm{Tn} 10$ in the $\mathrm{srl}$ rec $A$ region of the Escherichia coli $\mathrm{K}-12$ chromosome. Genetics 93, 321-343.

Fernandez, C., Larhammar, D., Servenius, B., Rask, L. \& Peterson, P. A. (1986). Spontaneous insertions into cosmid vector : a warning. Gene 42, 215-219.

Fishel, R. A., JAMES, A. A. \& Kolodner, R. (1981). rec A-independent general genetic recombination of plasmids. Nature, London 294, 184 186.

FRANKLIN, N. C. (1971). Illegitimate recombination. In The Bacteriophage Lambda, pp. 175-194. Edited by A. D. Hershey. Cold Spring Harbor, NY: Cold Spring Harbor Laboratory.

Hanahan, D. (1983). Studies on transformation of Escherichia coli with plasmids. Journal of Molecular Biology 166, 557-580.

Holmes, D. S. \& QuigleY, M. (1981). A rapid boiling method for the preparation of bacterial plasmids. Analytical Biochemistry 114, 193197.

Ish-Horowitz, D. \& BurKe, J. F. (1981). Rapid and efficient cosmid cloning. Nucleic Acids Research 9, 2989-2998.

Ishiura, M., OHASHI, H., UChIDA, T. \& OKaDA, Y. (1989a). Efficient simplified cosmid cloning: construction and characterization of cosmid vectors that carry the two cohesive end sites of $\lambda$ phages arrayed in tandem. Analytical Biochemistry 176, 117-127.

Ishiura, M., Hazumi, N., Koide, T., Uchida, T. \& OKada, Y. $(1989 b)$. A recB recC $s b c B$ recJ host prevents rec $A$-independent deletions in recombinant cosmid DNA propagated in Escherichia coli. Journal of Bacteriology 171, 1068-1079.

Jones, I. M., Primrose, S. \& Ehrlich, S. D. (1982). Recombination between short direct repeats in a recA host. Molecular and General Genetics 188, 486-489.

KUSHNER, S. R. (1978). An improved method for transformation of Escherichia coli with ColE1-derived plasmids. In Genetic Engineering, pp. 17-23. Edited by H. B. Boyer \& S. Nicosia. Amsterdam: Elsevier North-Holland.

LABAN, A. \& COHEN, A. (1981). Interplasmidic and intraplasmidic recombination in Escherichia coli K-12. Molecular and General Genetics 184, 200-207.

LEACH, D. R. F. \& STAHL, F. W. (1983). Viability of lambda phages carrying a perfect palindrome in the absence of recombination nucleases. Nature, London 305, 448-451.

LiLley, D. M. J. (1981). In vivo consequences of plasmid topology. Nature, London 292, 380-382.

LitTle, P. F. R. \& CRoss, S. H. (1985). A cosmid vector that facilitates restriction enzyme mapping. Proceedings of the National Academy of Sciences of the United States of America 82, 3159-3163.

LIU, L.-S. \& LARK, K. G. (1982). The Red function of phage lambda mediates the alteration of an interspersed repeated DNA sequence from the kangaroo rat Dipodomys ordii. Molecular and General Genetics 188, 27-36. 
Mahajan, S. K. (1988). Pathways of homologous recombination in Escherichia coli. In Genetic Recombination, pp. 118-121. Edited by R. Kucherlapati \& G. R. Smith. Washington, DC: American Society for Microbiology.

MaLoy, S. R. \& NunN, W. D. (1981). Selection for loss of tetracycline resistance by Escherichia coli. Journal of Bacteriology 145, 1110-1112.

Maniatis, T., Fritsch, E. F. \& SambrooK, J. (1982). Molecular Cloning, a Laboratory Manual. Cold Spring Harbor, NY: Cold Spring Harbor Laboratory.

Meselson, M. \& YuAN, R. (1968). DNA restriction enzyme from $E$. coli. Nature, London 217, 1110-1114.

MiLler, J. H. (1972). Experiments in Molecular Genetics. Cold Spring Harbor, NY: Cold Spring Harbor Laboratory.

Murray, N. E., Brammar, W. J. \& Murray, K. (1977). Lambdoid phages that simplify the recovery of in vitro recombinants. Molecular and General Genetics 150, 33-61.

Nader, W. F., Edlind, T. D., HuettermanN, A. \& Sauer, H. W. (1985). Cloning of Physarum actin sequences in an exonucleasedeficient bacterial host. Proceedings of the National Academy of Sciences of the United States of America 82, 2698-2702.

Saing, K. M., OriI, H., Tanaka, Y., Yanagisawa, K., Miura, A. \& IKEDA, H. (1988). Formation of deletion in Escherichia coli between direct repeats located in the long inverted repeats of a cellular slime mold plasmid: participation of DNA gyrase. Molecular and General Genetics 214, 1-5.

Sanger, F., Nicklen, S. \& Coulson, A. R. (1977). DNA sequencing with chain-terminating inhibitors. Proceedings of the National Academy of Sciences of the United States of America 74, 54635467.

ShEN, P. \& Huang, H. V. (1986). Homologous recombination in Escherichia coli: dependence on substrate length and homology. Genetics 112, 441-457.

Steinmetz, M., Winoto, A., Minard, K. \& Hood, L. (1982). Clusters of genes encoding mouse transplantation antigens. Cell 28, 489. 498.

Wyman, A. R., Wolfe, L. B. \& Botstein, D. (1985). Propagation of some human DNA sequences in bacteriophage $\lambda$ vectors requires mutant Escherichia coli hosts. Proceedings of the National Academy of Sciences of the United States of America 82, 2880-2884.

Yelton, M. M., Timberlake, W. E. \& van den Hondel, C. A. M. J. J. (1985). A cosmid for selecting genes by complementation in Aspergillus nidulans: selection of the developmentally regulated $y A$ locus. Proceedings of the National Academy of Sciences of the United States of America 82, 834-838. 